\title{
Multidimensional Association Rules on Tensors
}

Ryohei Yokobayashi, Takao Miura

Hosei University

Japan

ryohei.yokobayashi.2j@stu.hosei.ac.jp

miurat@hosei.ac.jp

ABSTRACT: In this work, we propose a framework suitable for multidimensional data mining based on tensor. A Tensor Data Model (TDM) provides us with high order data structure and naive description for information retrieval. Among others, we discuss multidimensional rule mining here. Generally, association rule mining (or extraction of association rules) concerns about co-related transaction records of single predicate, and hard to examine the ones over multiple predicates since it takes heavy time-and space-complexities. Here we show TDM allows us to model several operations specific to multidimensional data mining yet to reduce amount of description.

Keywords: Tensor Data Model, Multidimensional Association Rules, Data Mining

Received: 21 April 2018, Revised 28 May 2018, Accepted 3 June 2018

DOI: $10.6025 / \mathrm{jcl} / 2018 / 9 / 3 / 106-119$

(C) 2018 DLINE. All Rights Reserved

\section{Introduction}

Recently there happens a variety of sensor-devices and LSI memory and we may have many chances to access huge amount of the relevant information easily and quickly through internet. However, because of the wide variety of information, it is not easy to explore them consistently and efficiently. We hardly extract useful knowledge and these information disappear immediately very often.

Techniques to extract these knowledge have been investigated and proposed, they are called data mining. Because of its intuitiveness, association rule mining has been paid much attention[1]. Association rule mining concerns about co-related transaction records of single predicate, but few about multiple predicates. This research topic is called multidimensional association rule mining [3]. Clearly the larger dimensions we have, the more useful information we can extract[7]. That is, the problem gets hard about multiple predicates, because it takes heavy time- and space- complexities. For example, we may have usual association rules: Whenever A's stock goes up, so does B's. And we like to estimate that, whenever A's stock goes up, so does B's in a few days. In this case we like to extend the information by adding temporal information. To do that, we extend each stock price with timestamp so we have $(A, 10,180331)$ of 3 dimensional tensor structure "company, price, time".

106 International Journal of Computational Linguistics Research Volume 9 Number 3 September 2018 
In this investigation we introduce a Tensor Data Model (TDM)[13]. TDM is advantageous because we can model multiple aspects (in terms of ranks) from single view so that we can extract multi-dimensional association rules and also we can examine whether the processes and results are really useful or not in an intuitive manner. Several operators to TDM allow us to retrieve, manipulate, transform and modify the contents, Similar to relational algebra or OLAP operators including embedded operations. Here we define several operators for data mining on TDM as well as for data retrieval by which we can model them in an rather abstract manner.

This work contributes mainly to the following 3 points:

(1) We propose a collection of operators for data mining on TDM.

(2) We discuss how to extract multi-dimensional association rules on TDM.

(3) We examine the space complexity.

The rest of the paper is organized as follows. In section 2 we describe TDM, the data description and data manipulation. In section 3 , we discuss general frameworks of multidimensional data mining. Section 4 contains how to extract multi-dimensional association rules on TDM. . Section 5 contains an experimental results to see the effectiveness of this idea and we conclude this investigation in section 6 .

\section{Tensor Data Models}

\subsection{Describing High Order Data}

First of all let us define a tensor $X$ as a multidimensional array of data whose elements (or cells) are referred by multiple indices. The number of indices is called order of the tensor. Formally, given data structure $\mathcal{T}$ which is a real-values $\mathcal{R}$, we have a tensor $X$ of order $N$ as:

$$
X=\left\{X_{e_{1}, . ., e_{N}} \mid e_{j} \in I_{j}, j=1, \ldots, N\right\} \subseteq \mathcal{T}^{I_{1} \times I_{2} \times \ldots \times I_{N}}
$$

Here $X_{e 1}, \ldots,{ }_{e N}$ is a cell in $\mathcal{T}$ indexed by $e_{1}, \ldots, e_{N}, e_{j} \in I_{j}=\left\{1, \ldots,\left|I_{j}\right|\right\}$. The $k$-th index $I_{k}$ is called $k$-th dimension and $k$ is $m o d e$ of $X$. We also say $X \in \mathcal{T}\left[I_{1}, I_{2}, \ldots, I_{N}\right]$. Note there exists no duplicate value with same indices $e_{1}, \ldots, e_{N}$, that is, $X$ is functionally determined on $I_{1} \times I_{2} \times \ldots \times I_{N}$. A (conventional) vector is a tensor of order 1 and a matrix of order 2 . A high order tensor means the one of $N$ $\geq 3$ usually.

For instance, we show amount of monthly sales per item in a matrix format as in a figure 1 (left). A tensor $D$ of order 3 may contain amount of monthly sales per item and store as illustrated in a figure 1 (right). The tensor of order 3 is defined over STORE $\times$ ITEM $\times M O N T H$ where $S T O R E=\{$ store 1, store 2$\}, I T E M=\{$ item 1, item 2, item 3$\}$ and $M O N T H=\{$ January, July $\}$.

\subsection{Basic Operations for Tensor Data}

Let us introduce several operators over tensors[9][13]. We define projection, selection, add, subtract, product over tensors and multiply over tensor and matrix. Moreover, for the purpose of data mining, we introduce transform of orders, reduction and

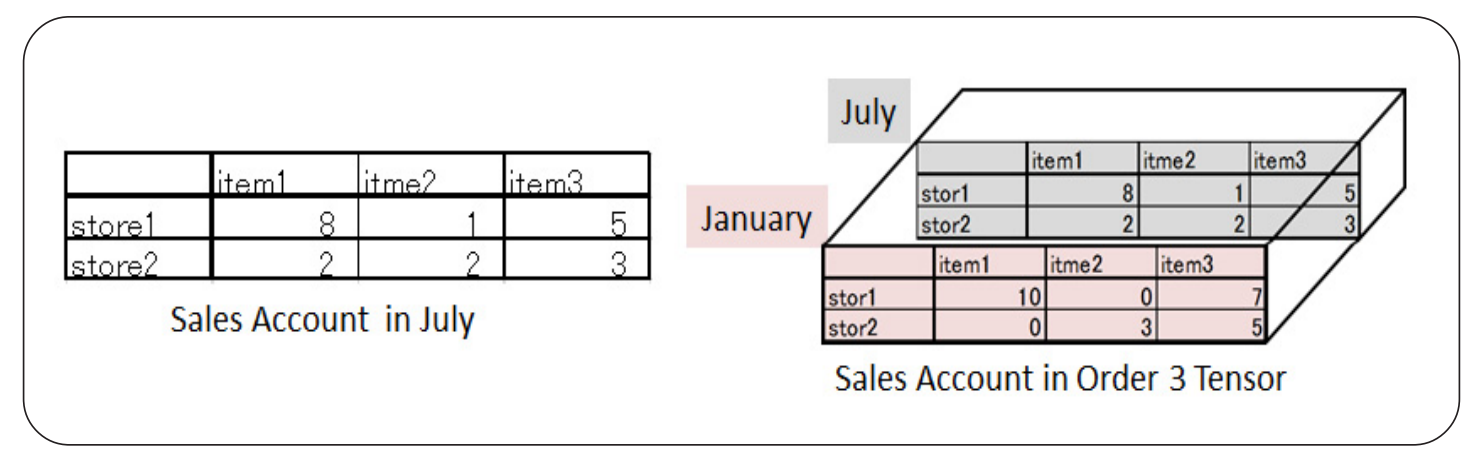

Figure 1. Amount of Sales 
recover of dimensions, vectorize and some other operators.

Let $X \in \mathcal{T}\left[I_{1}, I_{2}, \ldots, I_{N}\right]$. First we define two database operators, projection and selection. Let $A, B, . ., C$ be distinct modes or modes indexed by dimensions. Projection of $X$ over $A, B, \ldots, C$, denoted by $Y=\pi_{A, B, . .,}(X)$, is a subset of $X$ defined as :

$$
\begin{aligned}
\pi_{A, B, \ldots, C}(X)= & \left\{Z_{j_{1}, j_{2}, \ldots, j_{c}}\right\}, j_{1} \in A, j_{2} \in B, \ldots, j_{c} \in C \\
& Z_{j_{1}, ., j_{c}}=X_{i_{1}, \ldots, i_{N}}\left[j_{1}, ., j_{c}\right] \\
& \text { and } X_{i_{1}, . ., i_{N}} \in X
\end{aligned}
$$

Note $Y \in \mathcal{T}[A, B, \ldots, C]$ and that there might be several cells on $j_{1}, ., j_{c}$ in which case we can't define a tensor consistently

Selection of $X$ with conditions $C$, denoted by $Y=\sigma_{C}(x)$, is a subset of $X$ defined as :

$$
Y=\sigma_{C}(X)=\left\{X_{i_{1}, \ldots, i_{N}}\right\} \quad C\left(X_{1_{1}, \ldots, i_{N}}\right)=\text { true }
$$

Note that $Y \in \mathcal{T}\left[I_{1}, I_{2}, \ldots, I_{N}\right]$ and that the condition $C$ holds for $I_{1} \times \ldots \times I_{N}$. We might have an empty tensor if no index value satisfies the condition.

For example, we can obtain the matrix in the figure 1 (left) by $\sigma_{M O N T H}=$ "July" $(D)$. The combination provides us with a new tensor in the table 1 by $D_{3}=\pi_{\text {STOTE, ITEM }}\left(\sigma_{\text {MONTH }}=\right.$ "July" $\left.(D)\right)$. Let us note that $D_{1,2}=\pi_{\text {STOTE, ITEM }}(D)$ can't be well-defined as a tensor of order 2, because there exist 2 values, 10 and 8 , at $($ store 1 , item 1$) \in S T O T E \times I T E M$.

\begin{tabular}{|c|c|c|}
\hline & item1 & item2 \\
\hline store1 & 8 & 1 \\
\hline store2 & 2 & 2 \\
\hline
\end{tabular}

Table 1. Projection and Selection

Let $X, Y \in \mathcal{R}\left[I_{1}, I_{2}, \ldots, I_{N}\right]$ and we introduce numerical operators over tensors[9][13]. Let us define $X+Y(a d d)$ as an element-wise operation. We can define $X-Y($ subtract $), X * Y$ (multiply) and $X / Y($ divide $)$ in a similar manner:

$$
\begin{array}{ll}
X+Y & Z_{i_{1}, . ., i_{N}}=X_{i_{1}, . ., i_{N}}+Y_{i_{1}, . ., i_{N}} \\
X-Y & Z_{i_{1}, ., i_{N}}^{\prime}=X_{i_{1}, . ., i_{N}}-Y_{i_{1}, . ., i_{N}} \\
X * Y & Z_{i_{1}, . ., i_{N}}^{\prime}=X_{i_{1}, \ldots, i_{N}} * Y_{i_{1}, . ., i_{N}} \\
X / Y & Z_{i_{1}, . ., i_{N}}^{\prime}=X_{i_{1}, . ., i_{N}} / Y_{i_{1}, . ., i_{N}}
\end{array}
$$

We also introduce numerical functions Max, Min:

$$
\begin{aligned}
& \operatorname{Max}(X, Y) \quad Z_{i_{1}, \ldots, i_{N}}=\max \left(X_{i_{1}, . ., i_{N}}, Y_{i_{1}, . ., i_{N}}\right) \\
& \operatorname{Min}(X, Y) \quad Z_{i_{1}, . ., i_{N}}^{\prime}=\min \left(X_{i_{1}, . ., i_{N}}, Y_{i_{1}, \ldots, i_{N}}\right)
\end{aligned}
$$

Given $X, Y \in \mathcal{T}\left[I_{1}, I_{2}, \ldots, I_{N}\right]$, Inner Product $\langle X, Y\rangle$ (or, $\left.X \cdot Y\right)$ is defined recursively as a real-value over element-pairs:

$$
\langle X, Y\rangle=X \cdot Y=\sum_{i=1}^{I_{1}} \sum_{i=2}^{I_{2}} \ldots \sum_{i=N}^{I_{N}} X_{i_{1}, i_{2}, \ldots, i_{N}} \times Y_{i_{1}, i_{2}, \ldots, i_{N}}
$$

${ }^{1}$ Note null is different from “ 0 ” or blank, for instance.

108 International Journal of Computational Linguistics Research Volume 9 Number 3 September 2018 
Norm of $X, \| X||$, is defined as $\sqrt{X \cdot X}$. Note the inner product generates a multidimensional correlation over two tensors.

Let $x_{(j)} \in R^{I_{j}}, j=1, \ldots, N$ is a tensor of order 1 (or a vector), composition of $\left\{x_{(1)}, \ldots, x_{(N)}\right\}$, denoted by $x_{(1)} \otimes, \ldots, x_{(N)}$ is a tensor $X \in$ $R\left[I_{1}, \ldots, I_{N}\right]$ :

$$
X=x_{(1)} \otimes \ldots \otimes x_{(N)}
$$

Here $X_{i_{1} \ldots i_{N}} \in X$ is defined as $x_{(1) i_{1}} \times \ldots \times x_{(N) i_{N}}$. We call $X$ as a composed tensor of $x_{(j)} \in R^{I_{j}}, j=1, \ldots, N$.

If $X, Y$ have composed tensors such that $X=x_{(1)} \otimes \ldots \otimes x_{(N)}$ and $Y=y_{(1)} \otimes \ldots \otimes y_{(N)}$ respectively, we define Product (or, outer product) of tensors $X, Y$, denoted by $Z=X \otimes Y \in \mathcal{T}\left[I_{1}, I_{2}, \ldots, I_{N_{X} N_{Y}}\right]$, as follows:

$$
\begin{aligned}
& X \otimes Y=\left\{Z_{i_{1}, . ., i_{N_{X} N_{Y}}}\right\} \\
& Z_{i_{1}, \ldots, i_{N_{X} N_{Y}}}=x_{(1) i_{1}} \ldots x_{\left(N_{X}\right) i_{N_{X}}} y_{(1) i_{1} \ldots y_{\left(N_{Y}\right) i_{N_{Y}}}}
\end{aligned}
$$

For instance, let us define two tensors $X$ and $Y$.

$$
\begin{aligned}
& X=\sigma_{M O N T H}=" \text { January" }(D) \\
& Y=\sigma_{M O N T H}=" J u l y "(D)
\end{aligned}
$$

Then, the inner product $X \cdot Y$ means (extended) sales correlation of January and July.

As for relationship among a tensor $X$, a matrix $M$, a vector $V$ and a scalar $c$, let us consider a high order tensor $X$ as a collection of vectors, called $j$-th fiber, if we think of the tensor $X$ whose entries are fixed expect $I_{n}: X_{i 1}, \ldots, i_{n-1},{ }^{*}, i_{n+1}, \ldots, i_{N}$. A figure 2 contains 3 types of fibers given a tensor of order 3 along each mode. They are intuitively called mode- 1 fiber (column fiber, $n=1$ ), mode 2 (row fiber, $n=2$ ) and mode 3 (tube fiber, $n=3$ ) respectively.

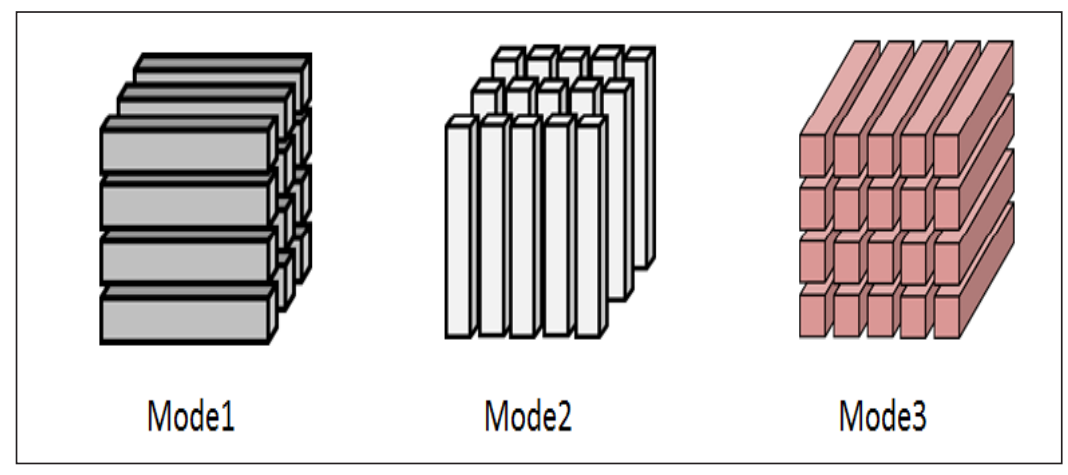

Figure 2. Fiber and Mode

For matricization of a tensor, we give the formalization by using mode-n fibers[9]. As illustrated in a figure 3, we consider the twodimensional slices where the horizontal slice (all the mode 1 fibers), lateral slice (all the mode 2 fibers) and frontal slice (all the mode 3 fibers) as matrices from a tensor of order $N$.

Let us define unfold or mode- $n$ matricization. $A$ tensor $X$ can be represented when we unfold the tensor into a matrix. The unfolding $X$ along mode $n$, denoted by $X_{(n)}$, is a matrix of dimension $I_{n} \times\left(I_{n+1} \ldots I_{N} I_{1} \ldots I_{n-1}\right)$ considering all the mode-n fibers as vectors. For example, each column of $X_{(n)}$ is a column of $X$ along the $n$-mode. Note the matrices are denoted by $X_{(1)}, \ldots, X_{(N)}$, of the size $I_{n} \times\left(I_{n+1} \ldots I_{N} I_{1} \ldots I_{n-1}\right), n=1,2, . ., N$.

For example, let us show examples of mode-1/2/3 matricizations in a table 2 applied to the figure 1.

International Journal of Computational Linguistics Research Volume 9 Number 3 September 2018 


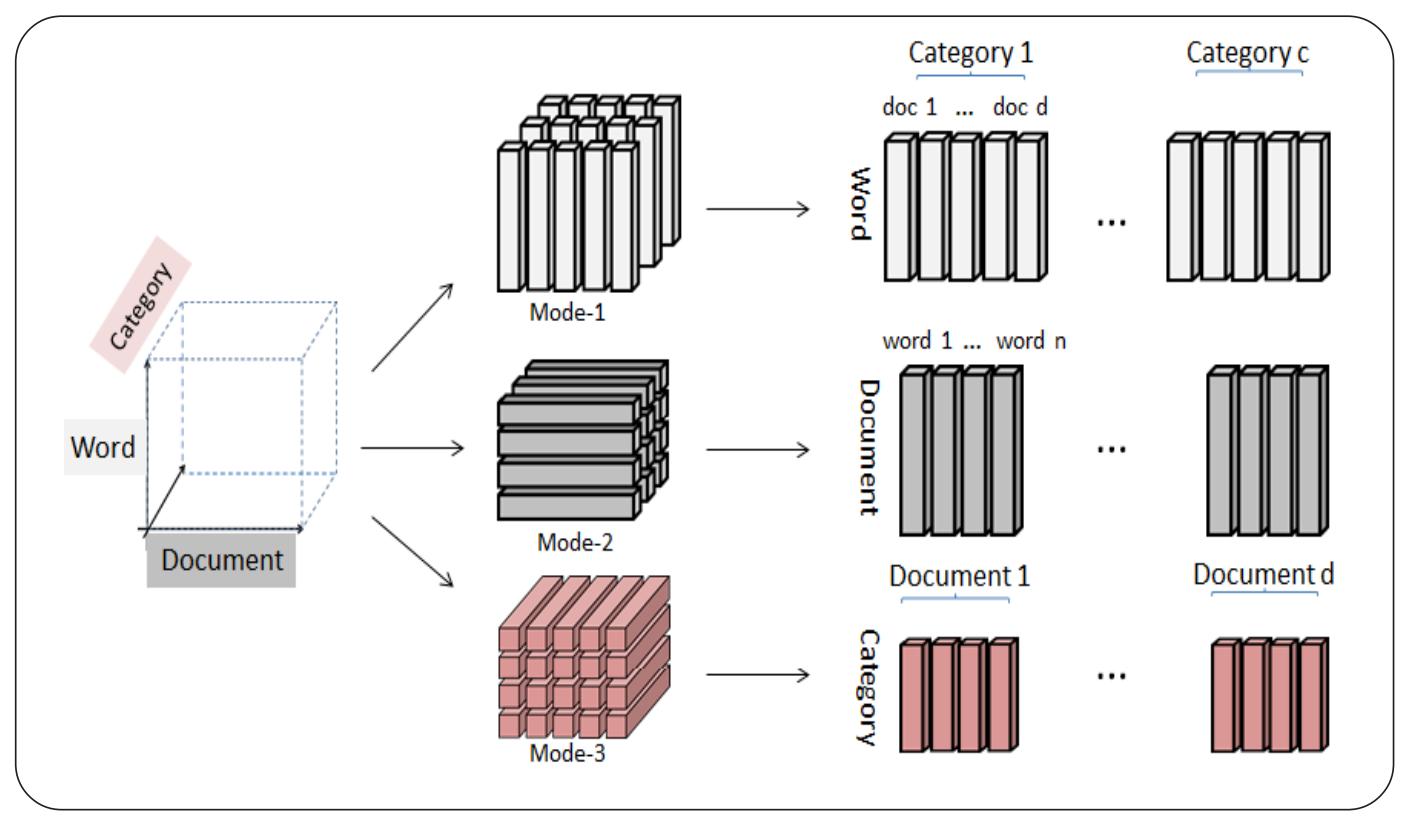

Figure 3. Matricization using Mode-n Fibers

\begin{tabular}{|c|c|c|c|c|c|c|}
\hline & Jan.item 1 & Jan.ite2 & Jan.ite3 & Jul.ite 1 & Jul.ite2 & Jul.ite3 \\
\hline storel & 10 & 0 & 7 & 8 & 1 & 5 \\
\hline store2 & 0 & 3 & 5 & 2 & 2 & 3 \\
\hline & st1.Jan & st1.Jul & \multicolumn{2}{|c|}{ st2.Jul } & & \\
\hline item1 & 10 & 8 & 0 & 2 & & \\
\hline item2 & 0 & 1 & 3 & 2 & & \\
\hline \multirow[t]{2}{*}{ item3 } & 7 & 5 & 5 & 3 & & \\
\hline & ite1.stl & ite1.st2 & ite2.st1 & ite2.st2 & ite3.st1 & ite3.st2 \\
\hline January & 10 & 0 & 0 & 3 & 7 & 5 \\
\hline July & 8 & 2 & 1 & 2 & 5 & 3 \\
\hline
\end{tabular}

Table 2. Mode- $n$ Matricization, $N=1,2,3$

Now let us define formally mode-n product or tensor-matrix multiplication. Given a tensor $X \in \mathcal{T}\left[I_{1}, I_{2}, \ldots, I_{N}\right]$ and a matrix $M \in$ $\mathcal{T}_{J n} \times I n$, we say $Y$ is a tensor of the mode-n product, $Y=X \times{ }_{n} M$ in $\mathcal{T}\left[I_{1}, I_{2}, ., I_{n-1}, J_{n}, I_{n+1}, \ldots, I_{N}\right]$ where $j_{n}=1, \ldots, J_{n}$ :

$$
\begin{aligned}
& X \times_{n} M=\left\{Y_{i_{1}, . ., i_{n-1}, j_{n}, i_{n+1}, . ., i_{N}}\right\} \\
& Y_{i_{1}, . ., i_{n-1}, j_{n}, i_{n+1}, . ., i_{N}} \\
& \quad=\sum_{i_{n}=1}^{I_{n}} X\left[i_{1}, . ., i_{n-1}, i_{n}, i_{n+1}, . ., i_{N}\right] M\left[j_{n}, i_{n}\right]
\end{aligned}
$$

By the definition we mean $Y(n)=M X(n)$ and also we have some properties:

$$
\begin{aligned}
& X \times_{m} A \times_{n} B=X \times_{n} B \times_{m} A, \quad m \neq n \\
& X \times_{n} A \times_{n} B=X \times_{n}(B A)
\end{aligned}
$$

One important special case is tensor-vector multiplication. Given a tensor $X \in \mathcal{T}\left[I_{1}, I_{2}, \ldots, I_{N}\right]$ and a vector (or one row matrix) $V$ $\in T_{I n}$, the multiplication, $Y=X \times{ }_{n} V$, is a tensor in $\mathcal{T}\left[I_{1}, I_{2}, ., I_{n-1}, I_{n+1}, . ., I_{N}\right]$ where 


$$
\begin{aligned}
& X \times_{n} V=\left(\left(Y_{i_{1}, . ., i_{n-1}, i_{n+1}, . ., i_{N}}\right)\right) \\
& \left.Y_{i_{1}, . ., i_{n-1}, i_{n+1}, . ., i_{N}}=\sum_{i_{n}=1}^{I_{n}} X\left[i_{1}, . ., i_{n-1}, i_{n}, i_{n+1}, . ., i_{N}\right] \cdot V\left[i_{n}\right]\right)
\end{aligned}
$$

This means $Y$ becomes one order smaller than $X$ where the $Y$ value at $i_{1}, \ldots, i_{n-1}, i_{n+1}, \ldots, i_{N}$ becomes inner-product with $V$. We also introduce a normalized multiplication $Y=X \times{ }_{n}^{0} V$, which means the multiplication normalized by the fiber lengths.

$$
\begin{aligned}
& X \times_{n}^{\circ} V=\left(\left(Y_{i_{1}, . ., i_{n-1}, i_{n+1}, . ., i_{N}}\right)\right) \\
& Y_{i_{1}, \ldots, i_{n-1}, i_{n+1}, . . i_{N}}= \\
& \quad\left(\sum_{i_{n}=1}^{I_{n}} X\left[i_{1}, . ., i_{n-1}, i_{n}, i_{n+1}, . ., i_{N}\right] \cdot V\left[i_{n}\right]\right) / L \\
& L=\sqrt{\left.\left(\sum_{i_{n}=1}^{I_{n}} X\left[i_{1}, . ., i_{n-1}, i_{n}, i_{n+1}, . ., i_{N}\right]\right)^{2}\right)\|V\|}
\end{aligned}
$$

A tensor-scalar multiplication can be defined in a straightforward manner. Given a tensor $X \in \mathcal{T}\left[I_{1}, I_{2}, \ldots, I_{N}\right]$ and a scalar $c \in R$, let us define $Z=c X$ as $Z_{i 1}, \ldots,{ }_{i N}=c X_{i 1}, \ldots,{ }_{i N}$.

For example, we multiply the tensor $D^{2 \times 3 \times 2}$ in the figure 1 by a matrix $M^{2 \times 3}$, and by a vector $V^{3}$ both in mode 2 :

$$
\begin{gathered}
D \times 2 M=D \times 2\left(\begin{array}{ccc}
1 & 3 & -1 \\
2 & -4 & 5
\end{array}\right)=\left(\begin{array}{cc|cc}
3 & 4 & 6 & 5 \\
55 & 13 & 37 & 11
\end{array}\right) \\
D \times 2 V=D \times 2\left(\begin{array}{ccc}
1 & 3 & -1
\end{array}\right)=\left(\begin{array}{ll}
3 & 4 \\
6 & 5
\end{array}\right)
\end{gathered}
$$

Table 3. Matrix/Vector Multiplication

\subsection{Data Mining on Tensor Data}

Here we introduce 2 operators, vectorize and shift, mainly for data mining on tensor, and count operations.

Each cell in a tensor may contain a real value so that often we need to transform this cell to a bit value to indicate a given cell is empty or not, and to keep a bit vector along one dimension.

To vectorize a tensor $X \in \mathcal{T}\left[I_{1} \times I_{2} \times \ldots \times I_{N}\right]$ where $I_{k}=\left\{1, \ldots,\left|I_{k}\right|\right\}$, we define $Y=(X)$ as $y_{i 1 \ldots i N}=1$ if $\left.x_{i 1} \ldots i_{N}\right) \neq 0,=0$ otherwise as illustrated in a figure 4 .

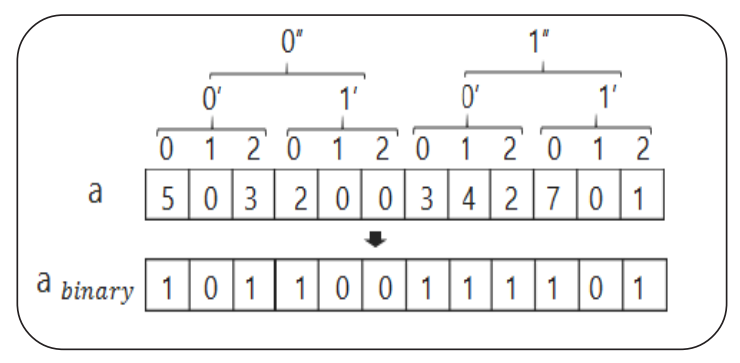

Figure 4. Vectorizing a tensor

A shift $(i)$ operator causes shifting elements along a dimension $i$ in a tensor $X$. That is, Shift operator causes one step shift to the right along $i$-th dimension as illustrated in a figure 5 .

$$
\begin{aligned}
Z=\operatorname{Shift}(X, i) & \pi_{i_{2}, \ldots, i_{N}}\left(z_{i_{1}, i_{2}, \ldots, i_{N}}\right)=\pi_{i_{2}, ., i_{N}}\left(x_{i_{1}, i_{2}, ., i_{N-1}}\right) \\
& =\pi_{i_{1}}\left(z_{i_{1}, i_{2}, \ldots, i_{N}}\right)=0
\end{aligned}
$$

Finally let us note that we can count all the elements using some combination of the operators. Let $Y$ be a vector $E$ where 


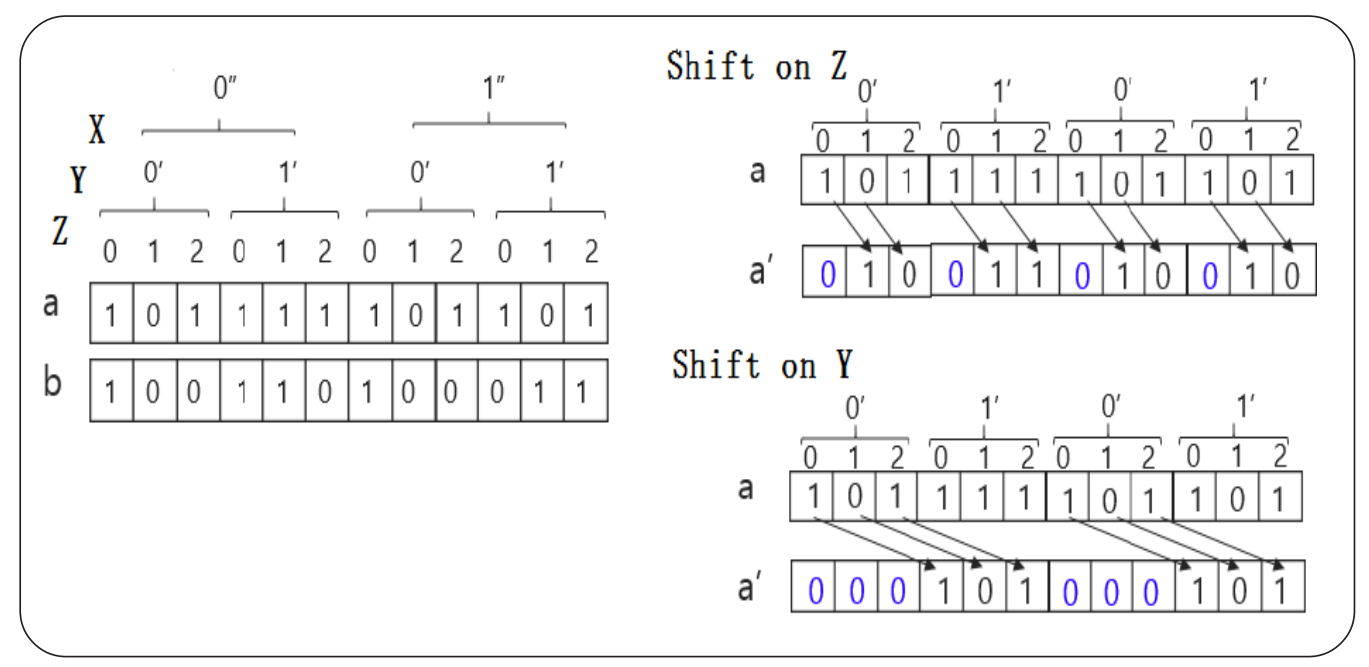

Figure 5. Tensot Shift

all the cells contain 1 . Given a tensor $X$ of order $1, X^{n \times m} \times Y^{m \times 1}=\left(Z^{n \times 1}\right)$ contains the number of " 1 " in $X$, or count, as illustrated below:

$$
\left[\begin{array}{lll}
1 & 1 & 1 \\
0 & 0 & 1
\end{array}\right] \times\left[\begin{array}{lll}
1 & 1 & 1
\end{array}\right]^{t}=\left[\begin{array}{l}
3 \\
1
\end{array}\right]
$$

\section{Extracting Association Rules on Tensors}

Market basket analysis is a typical example for big-data application such as sales information of supermarkets where we might consider transaction data (or an item set) as a unit of logical processing. Among others, an association rule has been put much attention so far, the rule means a significant conditional probability $P(X Y \mid X)$, denoted by $X \Rightarrow Y$.

After formalizing the issue, we show some extension of the approach for multidimensional sets of items and discuss why we get difficulties here.

\subsection{APRIORI}

In 1993, Agrawal[1] has proposed an effective algorithm named APRIORI, to obtain association rules from a collection $D$ of itemsets over items $\mathcal{I}=\left\{i_{1}, i_{2}, \cdots, i_{s}\right\}: D \subseteq 2^{\mathcal{I}}-\{\phi\}$. Let us show some example of transactions (and their itemsets) on the left of a table 4.

APRIORI algorithm allows us to extract association rules, denoted by $a \Rightarrow b$, which means, if any itemset contains an item $a$, the itemset also contains $b$, and if the itemsets containing both $a$ and $b$ appear frequently. Formally we define notions of support and confidence. Assuming there happen $N$ transactions, let $C(X)$ be the number of transactions containing $X$.

$$
\begin{array}{r}
\operatorname{support}(X)=\frac{C(X)}{N} \\
\operatorname{confidence}(Y \mid X)=\frac{C(X, Y)}{C(X)}
\end{array}
$$

Given two threshold values $\sigma$ and $\rho$ called minimum support and minimum confidence respectively, we extract all the pairs $X, Y$ of itemsets such that $\operatorname{support}(X, Y) \geq \rho$ and $\operatorname{confidence}(Y \mid X) \geq \rho$. Note that $X \subseteq Y$ means $C(X) \geq C(Y)$, called anti monotonicity. And APRIORI take an advantage of this property to avoid heavy computation. We compute $C(Y)$ only if all the $X \subseteq Y$ satisfy $C(X)$ $\geq \sigma$. Otherwise we don't need to examine it. Similarly, once we have $X \Rightarrow Y$ which means $C(X Y) / C(X) \geq \rho$, we must have $X \Rightarrow Z$ if $X Z \subseteq X Y$. This is because $C(X Z) / C(X) \geq C(X Y) / C(X) \geq \rho$.

\subsection{Multidimensional Association Rules}

There has been some investigation [7] proposed so far for the purpose of multidimensional association rule mining. In this

112 International Journal of Computational Linguistics Research Volume 9 Number 3 September 2018


subsection, we transform transactions into 2 dimensional data where we introduce a notion of distance to extract (2- dimensional) association rules. Let $\Delta$ and $\Delta^{\prime}$ be two points in the 2 dimensional space that are indexed by $v$ and $u$ respectively. The points are denoted also by $\langle v\rangle$ and $\langle u\rangle$. We define relative distance $\left(\Delta, \Delta^{\prime}\right)$ as $u-v$ to the two points. If we assume to start at an origin 0 , or $\langle 0\rangle$, we can think about relative distance of $\Delta$, denoted by $(0, \Delta)$, as $v$.

Let us assume that $\Delta$ contains an item $i_{k}$, denoted by $\Delta\left(i_{k}\right)$. Or we may assume $\Delta$ contains a transaction $T_{k}$, denoted by $\Delta\left(T_{k}\right)$. Finally we mean all the possible items by $I_{e}$ and all the possible itemsets by $D_{e}$.

$\mathrm{Lu}[7]$ introduces a notion of distance into transactions to extract temporal association rules as in a table 4 .

\begin{tabular}{|c|c|c|c|}
\hline Transaction & Items & (Extended) Transaction & (Extended) Items \\
\hline$T_{1}$ & a,b,c & $\Delta_{0}\left(T_{1}\right)$ & $\Delta_{0}(a), \Delta_{0}(b), \Delta_{0}(c)$ \\
$T_{2}$ & c,d,e & $\Delta_{1}\left(T_{2}\right)$ & $\Delta_{1}(c), \Delta_{1}(d), \Delta_{1}(e)$ \\
$T_{3}$ & a,b & $\Delta_{2}\left(T_{3}\right)$ & $\Delta_{2}(a), \Delta_{2}(b)$ \\
$T_{4}$ & a,b,c,e & $\Delta_{3}\left(T_{4}\right)$ & $\Delta_{3}(a), \Delta_{3}(b), \Delta_{3}(c), \Delta_{3}(e)$ \\
$T_{5}$ & b,c,d,e & $\Delta_{4}\left(T_{5}\right)$ & $\Delta_{4}(b), \Delta_{4}(c), \Delta_{4}(d), \Delta_{4}(e)$ \\
$T_{6}$ & a,b,e & $\Delta_{5}\left(T_{6}\right)$ & $\Delta_{5}(a), \Delta_{5}(b), \Delta_{5}(e)$ \\
\hline
\end{tabular}

Table 4. Transactions and Items

Starting at $\Delta_{0}$, we see, for instance, an item $a$ in $T_{1}$ (or exactly an extended item $\Delta_{0}(a)$ in $\Delta_{0}\left(T_{1}\right)$ ) with several distances $0,2,3$ and 5. Clearly it is hard to assume that each extended item preserves all the possible distances with its extended transaction.

To extract efficiently yet consistently distance relationship about extended items and transactions, they apply maxspan algorithm to this case[5]. The maxspan value $(m s)$ is a threshold which we should observe items and distance within the value. Let us note that we should extend notions of support and confidence of itemsets $X, Y$ :

$$
\begin{aligned}
& \text { support }^{\prime}=\frac{\text { Number of transactions within } m s \text { containing } X, Y}{\text { Number of total transactions within } m s} \\
& \text { confidence }^{\prime}=\frac{\text { Number of transactions within } m s \text { containing } X, Y}{\text { Number of transactions within } m s \text { containing } X}
\end{aligned}
$$

\begin{tabular}{|c|c|c|c|c|c|}
\hline$\Delta_{0}\left(t_{1}\right)$ & $\Delta_{1}\left(t_{2}\right)$ & $\Delta_{2}\left(t_{3}\right)$ & $\Delta_{3}\left(t_{4}\right)$ & $\Delta_{4}\left(t_{5}\right)$ & $\Delta_{5}\left(t_{6}\right)$ \\
\hline$a$ & ${ }^{c} \quad e$ & $a$ & $e$ & $\begin{array}{ll}b & e\end{array}$ & $a$ \\
\hline$c$ & $d$ & $b$ & $b$ & $c$ & e \\
\hline
\end{tabular}

Let us show E-Apriori [7] to extract 2-dimensional association rules in Algorithm1. Once we take counts on itemsets with each distance, we can obtain 1-itemsets which satisfy both of minimum support and minimum confidence.

Figure 6. Multidimensional Association Rules

Note that we don't obtain exact values of both support and confidence, because we assume maxspan $(m s)$ size on distance and ignore any transactions outsize the windows. For instance, in the figure 6 , we have an association rule

$$
\Delta_{0}(a), \Delta_{1}(c) \Rightarrow \Delta_{3}(e)
$$


Here the rule has support $=\frac{2}{5}$ and confidence $=\frac{2}{3}$. On the other hand, we must have support $=\frac{2}{5-3}$ and confidence $=\frac{2}{2}$ which depend on the span value $3=\left(\Delta_{3}-\Delta_{0}\right)$. In fact, we examine 3 windows $\left\{\Delta_{0}\left(T_{1}\right), \Delta_{1}\left(T_{2}\right), \Delta_{2}\left(T_{3}\right), \Delta_{3}\left(T_{4}\right)\right\},\left\{\Delta_{1}\left(T_{2}\right), \Delta_{2}\left(T_{3}\right), \Delta_{3}\left(T_{4}\right)\right.$, $\left.\Delta_{4}\left(T_{5}\right)\right\}$ and $\left\{\Delta_{3}\left(T_{4}\right), \Delta_{4}\left(T_{5}\right), \Delta_{5}\left(T_{6}\right)\right\}$.

Interestingly $\mathrm{Lu}$ [7] has shown that the true values could be approximated by the above support' and confidence' ${ }^{\prime}$, because there should happen huge number of transactions so that we must have few difference and anti-monotonicity.

To obtain 2-itemsets, we generate all the pairs of frequent 1 - items and all the items starting at $0^{\prime \prime} 0^{\prime} 0$ as in 1 -items. We take counts on an item with relative distances and obtain frequent ones using the minimum support/confidence. As for larger itemsets, we apply the above procedure to obtain frequent itemsets.

\section{Multidimensional Association Rule on Tensors}

In this section we discuss rule mining on (multidimensional) tensor data model, which can be seen as a natural generalization of $\mathrm{Lu}[7]$.

To apply rule mining on tensor, we need mode-n matricization, vectorize, shift and count operators/operations described previously.

We summarize the whole algorithm in an Algoritm 2. Let us who how our algorithm goes in the table IV. First we transform the table data by matricization in order to clarify relationship among items. We assume tensors of rank 4 for simplicity. To obtain all the 1 -item sets $\Delta_{0^{\prime \prime} 0^{\prime} 0}\left\{i_{n}\right\}, n=1, .$. , we take counts by inner-product of vectorized data and $a$ vector 1 and choose the vectors more than minimum support. That is, for the 1-itemset at $0^{\prime \prime} 0^{\prime} 0$, we take any other 1 -itemset $\left.\Delta_{s^{\prime \prime}} s^{\prime} s_{n}\left\{i_{n}\right\} s^{\prime \prime} s^{\prime} s \neq 00^{\prime \prime} 0^{\prime} 0\right)$, and apply (right) "shift" operator in such a way that $x^{\prime \prime}=s^{\prime \prime}, x^{\prime}=s^{\prime}, x=s$ and take count on the result as illustrated in a figure 7. After taking counts with all other itemsets and examining support and confidence values, we obtain frequent 1 -item sets.

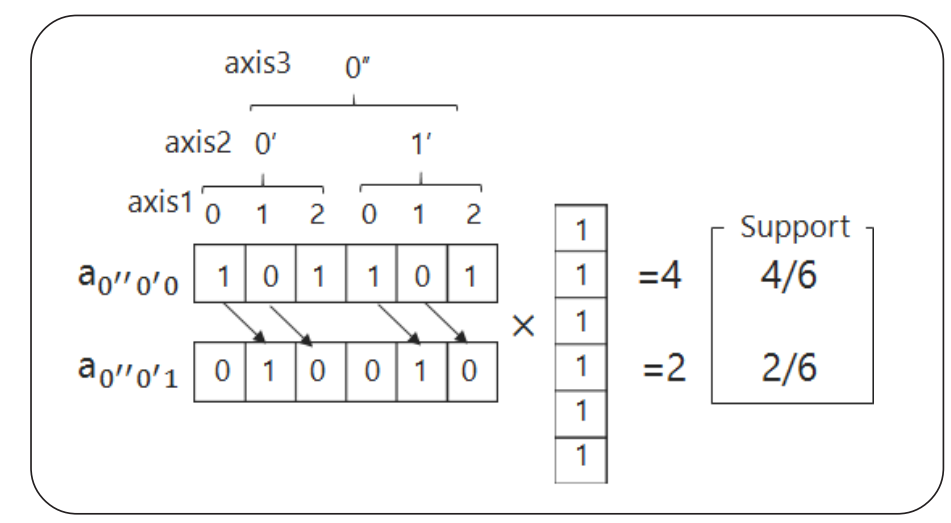

Figure 7. 1- itemsets

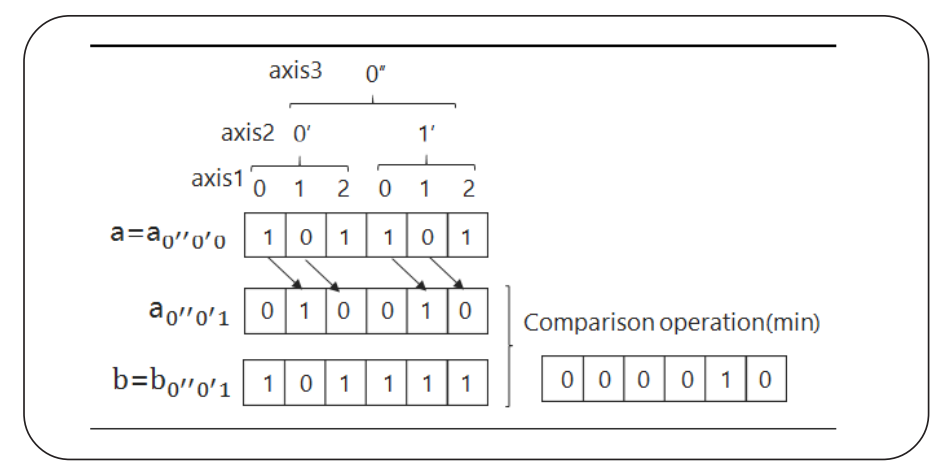

Figure 8. 2- itemsets 
As for 2 or more itemsets, let us generate $k$-itemsets $C_{k}$ from frequent $(k-1)$ itemsets $F_{k-1}$. For each $k$ - 1 itemset appeared in vectorized data $\Delta_{0^{\prime \prime} 0^{\prime} 0}\{a\}$ and $\Delta_{0^{\prime \prime} 0^{\prime} 1}\{a, b\}$, we apply (right) "shift" operator to the vector $a$ and $b$ to adjust a to $0^{\prime \prime} 0^{\prime} 1$ from $0^{\prime \prime}$ $0^{\prime} 0$, so that we can consider two vectors as the ones of same dimensions even if they have different distance, and extract association rules. See a figure 8 . Finally we examine both support and confidence by taking counts to obtain frequent itemsets.

\section{Experiments}

Here let us discuss some experimental results to show how well our approach works in terms of computing complexity and data description length compared to E-Apriori. We examine weather data in 2000 by meteorological satellite Himawari containing altitude, area and temporal information.

\subsection{Preliminaries}

In our experimental data Himawari 2000, all the information have been collected every day in 2000 through Automated Meteorolrgical Data Acquisition System $(A M e D A S)$. We take 3 axis, period, area and altitude with distance and construct a tensor of order 3.

Area consists of 8 regions: Hokkaido, Tohoku, Kanto, Hokuriku, Tokai, Kinki, ChugokuShikoku and Kyushu region. Altitude consists of 8 elevation sections where we divide altitude $0 \mathrm{~m}--240 \mathrm{~m}$ every $30 \mathrm{~m}$. We select observation points randomly from each section considered as transactions. Period means a time zone of April 1 to April 10. Given 7 as maxspan value, we examine 4 groups of April 1 to 7,2 to 8, 3 to 9 and 4 to 10 where each group satisfies maxspan threshold.

We examine attributes of items appeared in transactions: daily precipitation, sunshine hours, maximum temperature, minimum temperature, average wind speed and maximum wind speed. We describe these values by quantification as illustrated in a table 5 . Each transaction is a vector of 25 dimension where each dimension contains 1 (YES) or 0 (NO).

E-Apriori assumes single element in every attribute so that transactons have 6 attributes over YES/NO value.

As said previously, it takes heavy time and space complexities. Here we examine both time-complexity and space complexity to show TDM allows us to model several operations specific to multidimensional data mining yet to reduce amount of description. In this experiment, we compare our tensor approach and E-apriori with each other.

\begin{tabular}{|l|l|}
\hline daily precipitation $(\mathrm{mm})$ & $0,1 \sim$ \\
sunshine (hours) & $0 \sim 3,3 \sim 6,6 \sim 9,9 \sim$ \\
maximum temperature (deg.C) & $\sim 0,0 \sim 10,10 \sim 20,20 \sim 30,30 \sim$ \\
minimum temperature (deg.C) & $\sim 10,-10 \sim 0,0 \sim 10,10 \sim 20,20 \sim$ \\
average wind speed $(\mathrm{m} / \mathrm{s})$ & $0 \sim 3,3 \sim 6,6 \sim 9,9 \sim 12,12 \sim$ \\
maximum wind speed $(\mathrm{m} / \mathrm{s})$ & $0 \sim 3,3 \sim 6,6 \sim 9,9 \sim 12,12 \sim$ \\
\hline
\end{tabular}

Table 5. Quantification

\subsection{Results}

For association rule mining, it take same time complexity in both approaches. Assuming 0.80 and 0.50 as minimum confidence/ minimum support respectively, let us show how many rules we obtain in table1 6 and 7 .

We have extracted 1211 (multidimensional association) rules in total. Here are some example rules extracted:

maximum temperature $10 \sim 20$ in Kanazawa $\Rightarrow$ minimum temperature $\sim-10$ next day

maximum temperature $0 \sim 20$ in Wakkanai "and"

minimum temperature $\sim-10$ in Rokkasho $\Rightarrow$ minimum

temperature $\sim-10$ in Wakkanai next day

There happens no rule over more than 3 days and the attributes could be affected within 2 days in this case.

International Journal of Computational Linguistics Research Volume 9 Number 3 September 2018 


\begin{tabular}{c|c|c|c|c|c|c|c|c|c|c}
\hline No.Items & 1 & 2 & 3 & 4 & 5 & 6 & 7 & 8 & 9 & 10 \\
\hline $1 \sim 7$ & 93 & 422 & 919 & 926 & 463 & 153 & 44 & 10 & 0 & 0 \\
$2 \sim 8$ & 84 & 357 & 701 & 660 & 315 & 93 & 21 & 3 & 0 & 0 \\
$3 \sim 9$ & 83 & 393 & 821 & 808 & 409 & 148 & 58 & 24 & 7 & 1 \\
$4 \sim 10$ & 75 & 311 & 601 & 532 & 196 & 29 & 0 & 0 & 0 & 0 \\
\hline CommonItems & 73 & 267 & 442 & 335 & 109 & 15 & 0 & 0 & 0 & 0 \\
\hline
\end{tabular}

Table 6. Number Of Association Rules Per Itemset

\begin{tabular}{|l|c|}
\hline City & Number of Rules \\
\hline Sapporo & 671 \\
Hakodate & 633 \\
Akita & 200 \\
Sendai & 904 \\
Rokkasho & 671 \\
Tokyo & 113 \\
Yokohama & 240 \\
Kanazawa & 20 \\
\hline
\end{tabular}

Table 7. Number Of Association Rules Per City

\subsection{Discussion}

First of all let us show the data length of Himawari in a table 8. To compare description contents, we transform Eapriori algorithm into an algorithm 3 to generate efficiently more than 3 item sets. In our case, E-apriori requires space 549.8 times more.

\begin{tabular}{|c|c|c|c|}
\hline & Itemsets (all elements) & Relative Address & Total \\
\hline $\begin{array}{l}\text { E-apriori } \\
\text { Tensor }\end{array}$ & $\begin{aligned} 6 \times 8 \times 8 \times 7 & =2,688 \\
25 \times 8 \times 8 \times 7 & =11,200\end{aligned}$ & $6 \times \frac{(8 \times 8 \times 7)^{2}-1}{2}=602,109$ & $\begin{array}{c}604,792 \\
11,200\end{array}$ \\
\hline
\end{tabular}

Table 8. Data Description Length (Tensor and E-apriori)

Let us point out that Algorithm 2 has the part foreach (element $\Delta_{s^{\prime \prime} s^{\prime} s}(N)$ ) which requires time equal to the part foreach (extended transaction $\left.\Delta_{s^{\prime \prime} s^{\prime} s}(t)\right)$ in Algorithm 3, because both parts examine all the items in transactions. In other words, they take time of $O$ $\left(n^{3}\right)$.

To describe all the required data, we need the one for all the itemsets and for relative distancees so that our tensor model requires space $11,200 / 602,109=0.0186$ times (less than $2 \%$ ).

Important is a fact that, the more the data grows, the less ratio we have. The main reason comes from a fact that we need all the relative distances in E-apriori algorithm within maxspan range. More precisely, each order keeps (maxspan) of $N^{\prime \prime}, N^{\prime}, N$ relative distances, we should have the relative information $\left(N^{\prime \prime} \times N^{\prime} \times N\right)+\left(N^{\prime \prime} \times N^{\prime} \times N-1\right)+\ldots+1$. When there exist $M$ elements, we need space $M \times \frac{\left(N^{\prime \prime} \times N^{\prime} \times N\right)^{n-1}-1}{2}$ more for this purpose in E-apriori. Our tensor approach outperfoms whenever the amount of data grows.

Generally, the more orders the tensor holds, the more space we need dramatically for the relative distance in E-apriori. However, tensor description also requires more space in this case. For example, in E-apriori, each "sunshine hour" are described as one item 


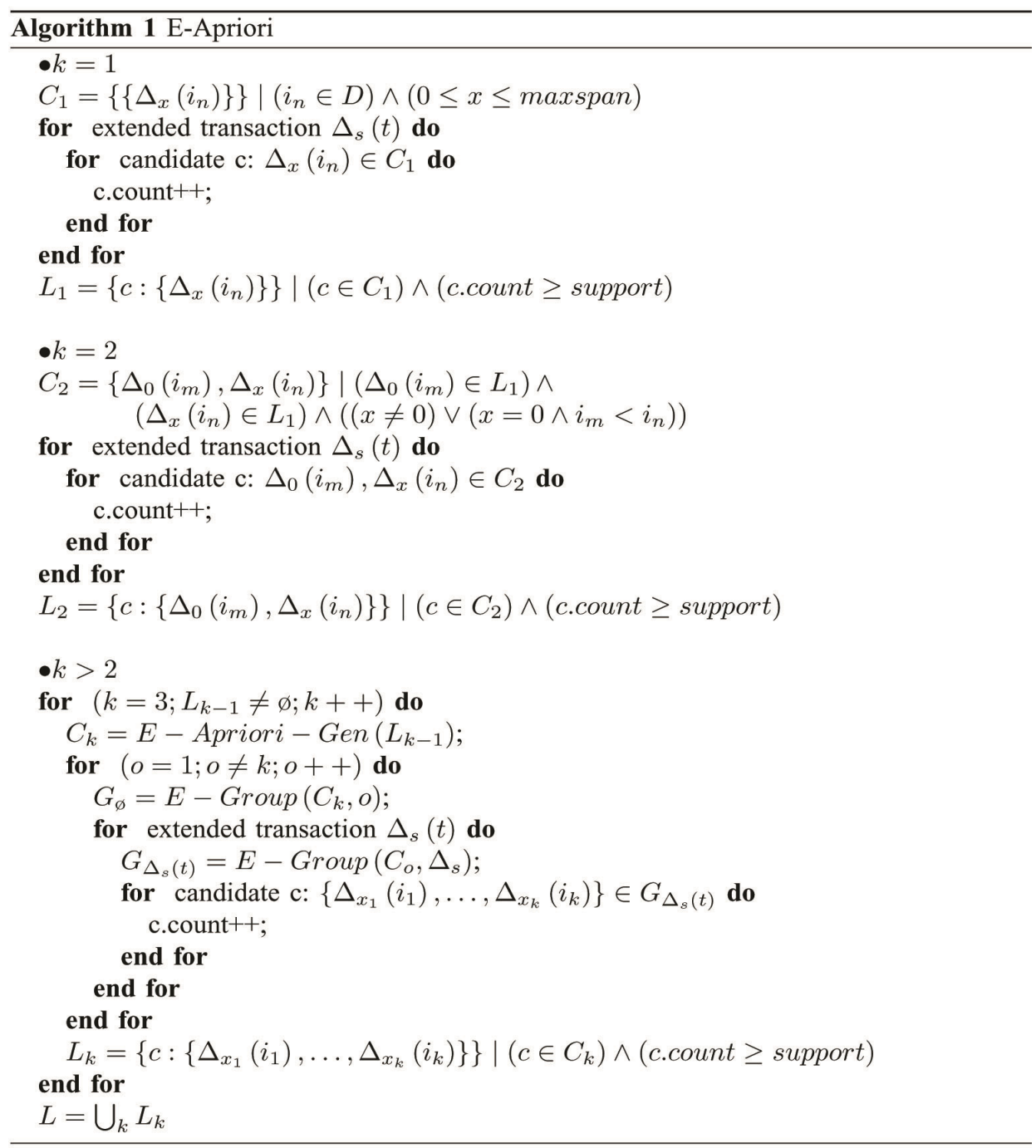

such as $0 \sim 3$ so that this kind of data becomes dense, while in tensor approach, we describe one sparce vector such as $(0 \sim 3,3 \sim 6$, $\because \ldots)$ and we must have more space.

\section{Conclusion}

In this investigation we have proposed a tensor data model for th epurpose of multidimensional association rule mining. We have introduced several operators such as vectorize and shift specific to the data mining operations. Then we have described a new algorithm within this framework and shown that simple algorithm works well based on the model. In our experiment, we examined weather data and extracted 1211 rules. Compared to a conventional E-apriori algorithm, we have shown that much less amount of space is enough for the data description.

\section{References}

[1] Agrawal, R., Imielinski, T., Swami, A. (1993). Database mining: A performance perspective, IEEE Transactions on Knowledge and Data Engineering 5 (6) 914-925.

[2] Gao, C., Wang, J. (2010). Direct mining of discriminative patterns for classifying uncertain data, In: Proceedings $16^{\text {th }}$ ACM SIGKDD International Conference on Knowledge Discovery and Data mining, $A C M, 2010$.

International Journal of Computational Linguistics Research Volume 9 Number 3 September 2018 

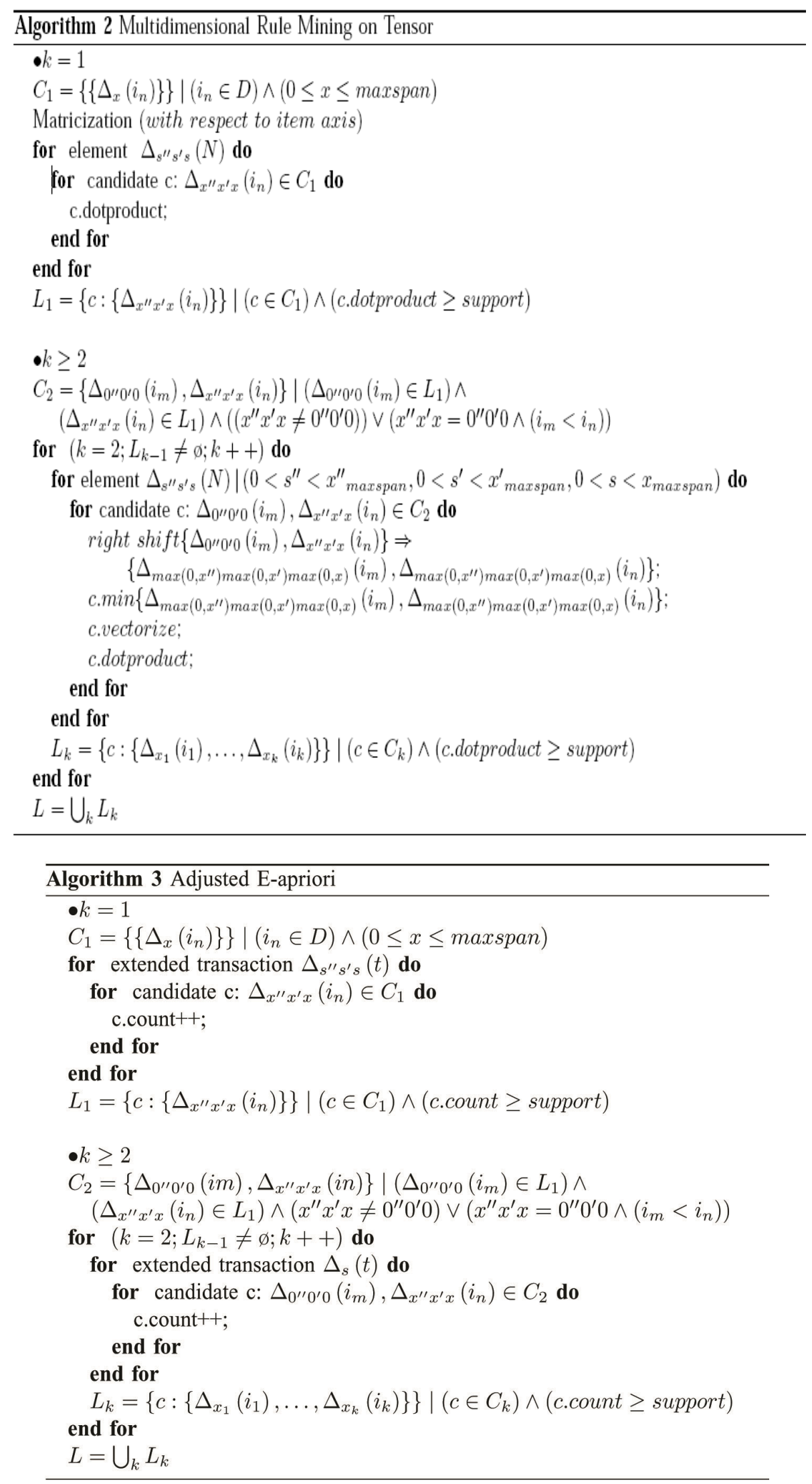
[3] Han, J., Pei, J., Kamber, M. (2011). Data mining: Concepts and techniques, Elsevier, 2011.

[4] Lieven, De L., Moor, B. De and Vandewalle, J. (2000). A Multilinear singular value decomposition, SIAM journal on Matrix Analysis and Applications 21 (4) 1253-1278.

[5] Lee, A. J. T., Wang, C-S., Weng, W-Y., Chen, Y-A., Wu, H-W. (2008). An efficient algorithm for mining closed inter-transaction itemsets, Data and Knowledge Engineering (DKE) 66, 68-91.

[6] Lu, H., Han, J., Feng, L. (1998). Stock movement prediction and dimensional inter-transaction association rules, 1998 ACM SIGMOD Workshop on Research Issues on Data Mining and Knowledge Discovery, Seattle, WA, USA, ACM, New York, USA.

[7] Lu, H., Feng, L., Han, J. (2000). Beyond intratransaction association analysis: mining multidimensional intertransaction associa tion rules, ACM Transactions on Information Systems (TOIS) 18 (4) 423- 454.

[8] Kaski, S. (1998). Dimensionality reduction by random mapping: Fast similarity computation for clustering, Neural Networks Proceedings, 1998. In: IEEE World Congress on Computational Intelligence. The 1998 IEEE International Joint Conference on. 1. IEEE.

[10] Nguyen, K.-N.T. et al. (2011). Multidimensional association rules in boolean tensors, In: Proceedings 2011 SIAM International Conference on Data Mining, Society for Industrial and Applied Mathematics.

[11] Sanguansat, P. (2010) Higher-Order Random Projection for Tensor Object Recognition, In: Intn'l Symp. on Communications and Information Technologies (ISCIT). IEEE, 2010.

[12] Sun, J., Tao, D., Faloutsos, C. (2006). Beyond streams and graphs: dynamic tensor analysis, In: Proceedings $12^{\text {th }}$ ACM SIGKDD international conference on Knowledge discovery and data mining. ACM.

[13] Yokobayashi, R. and Miura, T. (2017). Modeling random projection for tensor objects, In: Proceedings International Conference on Web Intelligence (WI), ACM. 